\title{
Transferts de chaleur et problèmes de circulation dans les échangeurs: revue de quelques progrès récents
}

\section{Heat transfer and problems related to circulation in heat exchangers: some recent developments}

\author{
P.AR M. TOUTY
}

INGENISUR A YA SOCHTE INDATOM

L'autenr résnme briènement et commente huit articles récemment parus :

P. Miller, J. Byrnes, D. Benforado : Heat transfer to water in an annulus. (Am. Inst. Chem. Eng. Journal, 1/4 dec. 1955, pp. 501-504).

P. Miller, J. Burnes, D. Benforado : Hcat transfer to water in a rod bundle. (Nuclear Engineering and Science Congress, Cleveland; déc. 12-16 1955-preprint n" 47.)

J. F. Mumu :

Heat transfer to boiling water forced through an electrically heated tube.

(Reactor heat transfer Symposium, Brookheaven National Laboratory, sept. 30, 1954.)

C. D. Alstad, H.S. Isbin, N. R. Amunuson,

J.P. SIIvVER :

Transient behavior of single phase, natural circulation loop.

(A.I.Ch.E. Journal 1/4, dec. 1955, pp. 417425.)
The Author briefly summarizes and comments eight recently issued papers:

E. H. Wissler, H.S. Ismin, N. R. AMUNDSON : The oscillatory behavior of a two phase, natural circulation loop.

(Nucl. Eng. Sc. Congress, Cleveland, dec. 12-16 1955, preprint 59.)

O. G. Hamman, IR. JUNG :

The effect of decreasing boiler pressure on natural circulation in water tube boilers. (Combustion, sept. 1955, pp. 47-55.)

M. W. Carbon, W. D. Gimisn't, C. R. Mac Nutt, R. Newer :

Steam generation in a reactor.

(Nuct. Sc. Eng. Congress, Cleveland; déc. 1955, preprint 46.)

A. R. Grunen, S. C. Hyman :

Flow distribution among parallel heated channels.

(Nucl. Sc. Eng. Congress, Cleveland, dec. 1955, preprint 349.) 
J'ai eu, depuis plusieurs mois, à me préoccuper des problèmes d'échanges de chaleur dans les réacteurs nucléaires et j'ai été, sur ce sujet, amené à dépouiller une documentation importante. Il m'a été demandé d'essayer aujourd'hui de vous faire partager l'expérience, toute livresque, que j'ai pu ainsi acquérir.

Le nombre des publications récentes relatives aux problèmes d'échanges thermiques est véritablement impressionnant. Une revue bibliographique systématique, dans le cadre d'un exposé de durée raisonnable, aboutirait à une sèche énumération de références, sans grand intérêt.

J'ai donc préféré sélectionner quelques mémoires seulement, qui m'ont paru apporter des résultats ou des idées nouvelles, et essayer d'en tirer l'essentiel.

D'autre part, la mécanique des fluides, et la science des échanges de chaleur, sont pour moi des sujets encore bien neufs, et je demande aux hydrauliciens et aux thermiciens chevronnés qui remplissent cette salle toute leur indulgence lorsqu'il m'arrivera, sur la foi de ce que j'ai lu, d'escamoter une difficulté ou, au contraire, tel monsieur de la Palisse, de mettre une ardeur déplacée à enfoncer des portes déjà grandes ouvertes.

Tout d'abord, sur la détermination des coefficients d'échange thermique, j'ai retenu trois mémoires. Deux d'entre eux ont été écrits par le même groupe d'auteurs :

P. Miller, J. Byrnes, D. Benforado:

Heat transfer to water in an annulus. American Institute of Chemical Engineers Journal, vol, $1, \mathrm{n}^{\circ}$ 4, Dec. 1955, pp. 501504.

Heat transfer to water in a rod bundle. Nuclear engineering and science congress sponsored by engineers joint council, Cleveland, Dec. 12-16, 1955, Preprint $n^{\circ} 47$.

Il s'agit, dans ces deux mémoires, d'essais effectués aux «Walter Kidde Nuclear Laboratories » à New-York. Dans les chaudières et échangeur's classiques, on est amené à s'intéresser aux échanges de chaleur entre paroi et eau, soit à l'intérieur de tuyaux circulaires, soit à l'extérieur d'un faisceau tubulaire, mais avec un écoulemenl plus ou moins perpendiculaire au faisceau. Ici, au contraire, il s'agit d'écoulements entre deux parois circulaires concentriques, ainsi que d'écoulements extérieurs parallèles à un faisceau tubulaire.

Les deux cas ainsi étudiés ne sont pas entièrement nouveaux, mais ne semblent pas avoir fait l'objet jusqu'à présent d'études expérimentales bien systématiques.

L'appareillage d'essais est à chauflage électrique par résistances. La surface d'échange est celle d'un barreau en aluminium chauffé inté- rieurement au moyen d'un ruban de nichrome enroulé en hélice sur un mandrin réfractaire. La résistance chauffante est alimentée en courant continu. La longueur de la partie chauffée est normalement de $10 \mathrm{~cm}$, mais atteint $20 \mathrm{~cm}$ sur un barreau utilisé seulement dans une partie des essais.

La principale difficulté, avec ce type d'appareillage, est d'obtenir' une bonne jonction thermique entre la résistance chaufrante et son enveloppe métallique, dont elle est électriquement isolée. Cette jonction est assurée ici par un enduit réfractaire. La conduction thermique de cet enduit est relativement faible; aussi, malgré les précautions prises pour assurer un centrage correct de la résistance chauffante, les résultats obtenus montrent qu'une certaine dissymétrie n'a pu être complètement évitée.

La première cellule d'essais, à écoulement annulaire, comprend un seul barreau placé au centre d'un tube de verre, ce qui permet d'observer l'écoulement. Le nombre de Reynolds variait entre 5.000 et 22.000 , et les essais ont été faits au voisinage de la pression atmosphérique.

Dans le deuxième montage, la barre étudiée est substituée à l'une ou l'autre des barres d'un faisceau fixe de symétrie hexagonale, placé à l'intérieur d'une enveloppe cylindrique. Le faisceau comprend au total 27 barres. Le nombre de Reynolds variait de 70.000 à 700.000 , et l'ensemble a été utilisé jusqu'à $10 \mathrm{~kg} / \mathrm{cm}^{2}$ et $163^{\circ} \mathrm{C}$. Comme les essais sont faits en vue de la construction de réacteurs nucléaires, il y a interêt à pousser les flux thermiques aussi haut que possible. Ici, la limitation provient de la température maximum admissible du ruban de nichrome. Le revêtement réfractaire cause une chute de température très importante, ce qui limite finalement le flux à 60 watts $/ \mathrm{cm}^{2}$. Ceci pour des températures d'eau de l'ordre de 20 à $50{ }^{\circ} \mathrm{C}$.

La plus grosse difficulté, du point de vue expérimental, est la mesure correcte de la température de paroi. Les auteurs utilisent des couples thermoélectriques dont la soudure est noyée dans la paroi d'aluminium, le plus près possible de la surface extérieure. L'amenée des fils se fait soit par l'extérieur (ils sont alors logés dans des rainures), soit par l'intérieur du barreau. Bien que le gradient de température - facile à calculer - soit relativement faible dans une paroi d'aluminium, les indications des différents thermocouples ne sont pas entièrement concordantes, à cause de l'incertitude inévitable sur la position exacte de la soudure.

Voyons maintenant les résultats obtenus : on porte, en coordonnées logarithmiques, en abscisses le nombre de Reynolds et en ordonnées le nombre de Nusselt. Les points expérimentaux se groupent le long d'une droite de pente 0,8 , ce 
qui est normal. Le résultat inattendu, e'est la valeur de l'ordonnée à l'origine.

Dans la première série d'expériences, en écoulement annulaire, le coefficient de convection est supérieur de $20 \%$ à celui que l'on calcule avec la formule classique de Colburn, établie pour l'écoulement dans un tube circulaire, en y remplaçant le diamètre du tube par le diamètre hydraulique correspondant à l'écoulement annulaire. Dans le deuxième cas, avec le faisceau cylindrique, la différence est encore plus grande, de l'ordre de $50 \%$ : au lieu de la formule de Colburn :

$$
\text { Ne }=0,023(\mathrm{Re})^{0,8}(\mathrm{Pr})^{0,3}
$$

les résultats obtenus vérifient la formule :

$$
\mathrm{Ne}=0,032(\mathrm{Re})^{0,8}(\mathrm{Pr})^{0,3}
$$

Ceci n'est pas entièrement surprenant : l'empoi de la formule de Colburn pour des écoulements cylindriques autres que dans un tuyau de scction circulaire n'est guère justifié en théorie, el les résultats obtenus ici tendraient à prouver que la notion de diamètre hydraulique ne suffit pas pour caractériser complètement la géométrie d'un écoulement de seclion non circulaire.

I.es auteurs ont également ćtudic les pertes de charge. On trouve bien que le coefficient de pertes de charge par frottement à la paroi est proportionnel à $(\mathrm{Re})^{0,2}$, mais le coefficient obtenu est 0,074 , supérieur de $65 \%$ aux mesures classiques obtenues dans des tubes.

On est évidemment tenté d'attribuer ces deux différences à une même cause, la rugosité des parois. Mais les auteurs insistent sur le fait (qu'ils ont utilisé des barreaux parfaitement lisses; lappareillage a été fréquemment démonté pour contrôler l'absence de toute rugosité due à l'encrassement ou l'érosion. Sous réserve que les mesures de température soient correctes, Ia seule hypothèse permettant raisonnablement d'expliquer les écarts obtenus est que, même si l'on admet qu'il suffit d'un paramètre pour définir le comportement hydraulique et thermique d'écoulements non géométriquement semblables, ce parametre est une fonction des dimensions plus compliquéc que le diamètre hydraulicue classique.

Je passe maintenant à un troisiène mémoire sur les coefficients d'ćchange :

\section{J. F. Mumm :}

Heat transfer to boiling water forced through an electrically heated tube (Reaclor Heat Transfer Symposium, Brookhaven National Laboratory, Sept. 30, '1954).

L'auteur appartient à la Compagnie Babcock et Wilcox américaine, mais le travail a été effectué pour le compte du laboratoire d'Argonne.
L'appareil comprend un tube horizontal de $11,6 \mathrm{~mm}$ de diamètre inlérieur, alimenlé en circulation forcée avec une vitesse massique de l'ordre de 30 à $135 \mathrm{~g} / \mathrm{cm}^{2}$, sous des pressions pouvant varier entre 2 et 13 atmosphères. Ie tube, à l'intérieur duquel circule l'émulsion eauvapeur, est chauffé par passage direct de courant alternatif. L'intensité nécessaire est obtenue en couplant en parallèle trois transformateurs de soudure. Ia longueur utile est de 1,5 m. Le flux thermique maximum est un peu plus élevé que dans les expériences précédentes, et peut atteindre 70 watts $/ \mathrm{cm}^{2}$. Le titre en vapeur à la sortie peut monter jusqu'à $60 \%$.

La mesure des températures de paroi pose beaucoup moins de problèmes que dans le cas précédent : il suffit de placer des thermocouples à la surface extérieure du tube, sous un calorifuge. La région où ils se trouvent placés est sensiblement isotherme, et la position exacte des soudures sans importance. On obtient ainsi la lempérature extérieure du tube. La lempérature intérieure s'en déduil aisément, car la chute de température à travers la paroi est relalivement faible, et peut être calculéc.

La mémoire ne donne que très peu de délails sur les résultats obtenus : ceux-ci sont en accord avec la formule :

$\mathrm{Ne}=\left[4,3+5 \cdot 10^{-4}\left(\frac{\mathrm{V}_{q}-\mathrm{V}_{0}}{\mathrm{~V}_{e}}\right)^{1,04} x\right]\left(\frac{q}{\mathrm{G} \lambda}\right)^{0,464} \mathrm{Re}{ }^{0,808}$

tant que $x$ reste inf'rieur à $40 \%$. Dans cette formule, $V_{a}$ et $V_{o}$ sont les volumes spécifiques respectifs de la vapeur et de l'eau, $x$ le titre de l'émulsion, q le fux thermique, $G$ la vilesse massique ef 7 la chaleur de vaporisalion. Ie long du tube, $x$ augmente régulièrement de l'entrée à la sortie. Fin même temps, le coefficient d'échange croit tout d'abord, passe par un maximum correspondant à $x==50 \%$, puis décroît rapidement.

Je passe maintenant à une autre sćric d'arlicles, consacres aux problèmes d'instabilité d'écoulement en présence d'un flux thermique:

(..). Alsma, H.S. Isisin, N, R. Amenoson, J. P. SHVERS :

Transient Behavios of single phase, natural circulation Joop systems. - A.I.CH.E. Journal, 1, 4, pp. 417-425 (Dec. 1955).

E. H. WISSLER, II. S. Isisin, N. R. AMUNHSON : The oseillatory behaviof of a two-phase natural circulation loop (Naclear Engineering and Science Congress, develand, 12-16 Dec. 1955, preprint 59).

L'appareillage utilisé est le même dans les deux cas : il s'asit d'une boucle à circulation naturelle. On a un dispositif de chauffage électrique à la base d'un tube ascendant; en haut 
de ce tube, un échangeur de refroidissement. Un tube descendant complete le circuit, qui est équipé d'organes de mesure du débit, ainsi que de la température en différents points. Une série d'expériences préliminaires, qui ne sont pas décrites en détail, permet de déterminer les caracréristiques hydrauliques et thermiques du système: coefficients de perte de charge, de convection, déperditions calorifiques des parois, inerties thermiques, etc.

Dàns le premier de ces deux mémoires, le comportement de la boucle, en eau, en régime transitoire, est étudié théoriquement et expérimentalement. C'est une tentative assez audacieuse, rui jusqu'à présent n'a guère eu d'équivalent dans la littérature. La mise en équation aboutit évidemment à un système intégro-différèntiel dont il n'y a pas grand-chose à tirer par les méthodes analytiques classiques. Mais il est toujours possible de décomposer la boucle en un nombre assez grand de sections élémentaires, et de remplacer le système intẻgro-différentiel initial par un système d'équations aux différences finies. Le calcul numérique des solutions est alors abordable, si l'on dispose d'une machine clectronique.

Dans les calculs ainsi effectucs, les coefficients de convection entre paroi et eau ont été supposés les mêmes en régime transitoire qu'en régime permanent. Cette approximation s'avère légitime, car les transitoires auxquels on abou- fonction du temps, la courbe du haut donne le débit, et les courbes suivantes la température en trois points du circuit. Ces grandeurs ont des oscillations très amorties, avant de se stabiliser à un nouveau régime permanent. La comparaison entre théorie et expérience s'avère très satisfaisante.

Dans le deuxième mémoire, l'appareillage utilisé est le même, mais la puissance de chauffe est augmentée jusqu'à obtenir la formation de vapeur dans le tube ascendant. Cette fois, au lieu d'avoir simplement des oscillations amorties après une perturbation, on observe couramment l'amorcage d'oscillations entretenues (fig. 2).

L'accord entre le calcul et les résultats d'expérience est un peu moins bon qu'en phase homogène: la période calculée diflère jusqu'à $20 \%$ de la période mesurée. Mais le calcul permet de prédire correctement la forme des oscillations, et je considère l'accord ainsi oblenu comme tout ¿̀ fait remarquable, étant donné la complexité du probleme. Le calcul met en relief les points suivants :

L'instabilité n'est oblenue que si la diminution de densité du fluide chauffé, qui fournit la pression motrice et entretient l'écoulement, agit, non seulement dans la partie chauf'ce directement, mais aussi le Jong de la colonne monlante qui Iui fait suite. Il faul aussi que l'augmentation relative de volume, rapportée à l'augmentation d'en-

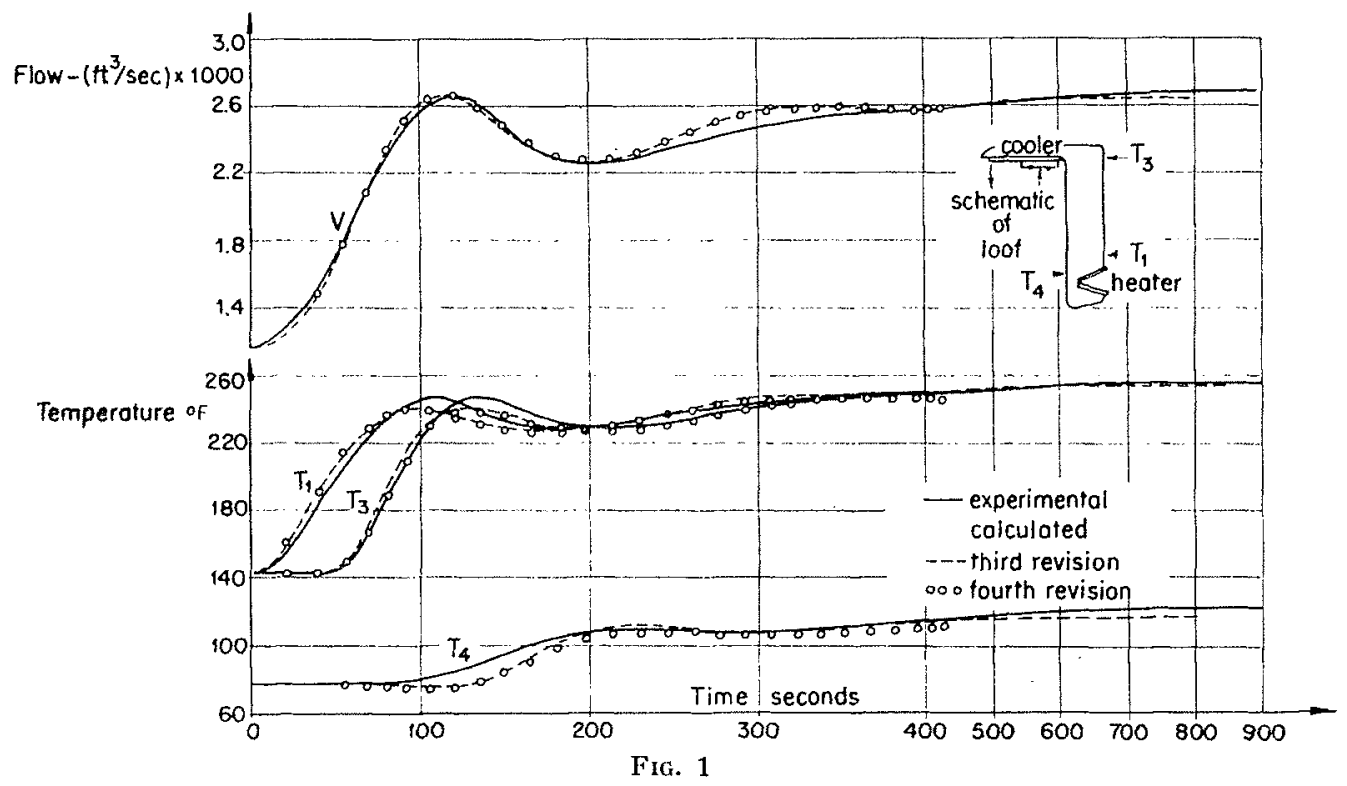

tit sont relativement lents, avec des constantes de temps de l'ordre de la minute.

La figure 1 ci-contre donne le résultat des calculs (en pointillé) comparé à l'expérience (trait plein) lors de transitoires consécutifs à une variation subite de la puissance de chauffe. En thalpie, par unité de masse du fluide, et multipliée par la hauteur de la colonne montante, atteigne une valeur suffisante.

Enfin, la période d'oscillation est du même ordre de grandeur que le temps de transit du fluide à travers la colonne montante. 


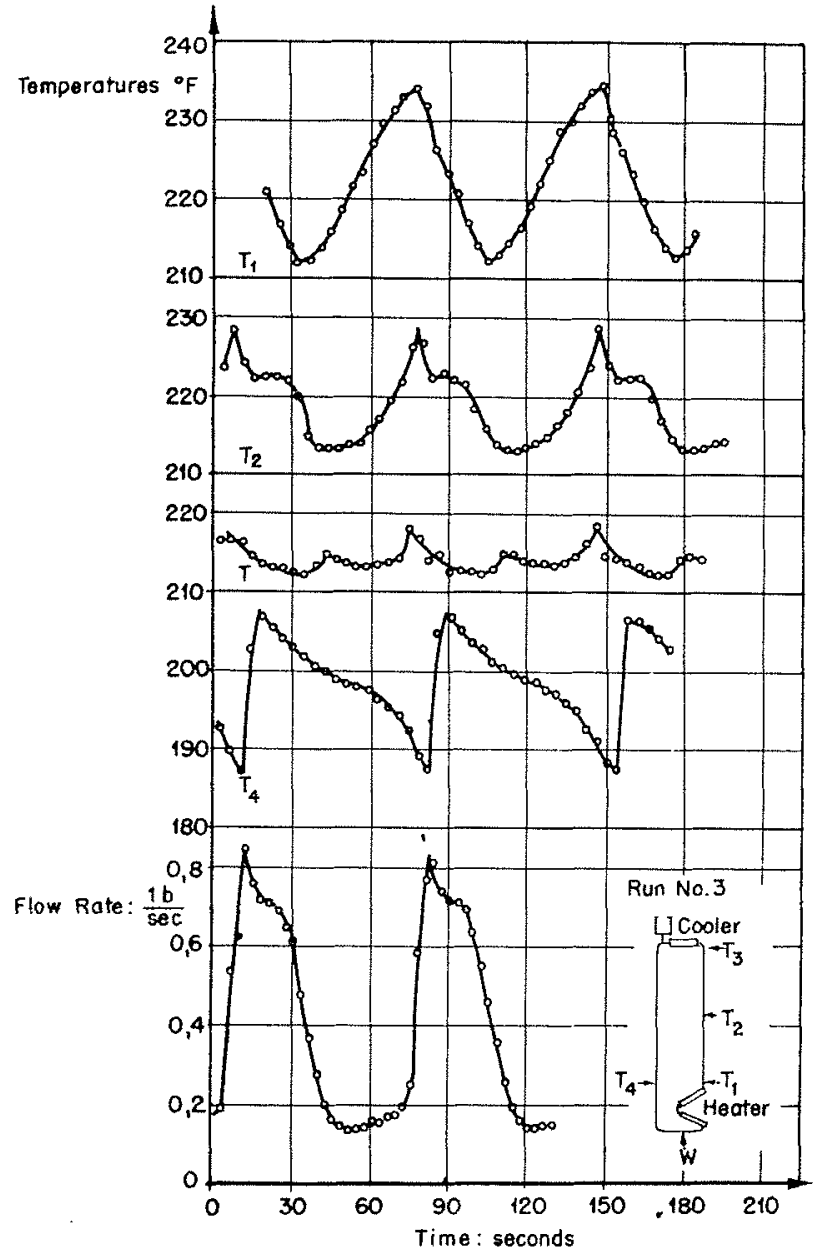

FIG. 2

A ces deux articles, j'en comparerai un troisième, qui traite d'un problème analogue, à l'échelle d'une chaudière industrielle :

\section{O. G. Hammár et R. Jung:}

The effect of decreasing boiler pressure on natural circulation in water tube boilers. - Combustion, Sept. 1955 , pp. 47 à 55 .

Il s'agit donc du comportement de la circulation naturelle dans une chaudière, en présence d'une baisse plus ou moins brusque de pression, provoquée par un accroissement de la demande de vapeur. Cette baisse de pression provoque normalement l'éclatement en vapeur de l'eau contenue dans le ballon supérieur, ainsi que l'augmentation du titre en vapeur de l'émulsion qui arrive en haut des tubes ascendants. Si elle est suffisamment rapide, elle provoque également la formation de vapeur dans les tubes de retour, ce qui freine la circulation. Après un régime transitoire plus ou moins long. comportant ou non l'augmentation de la puissance de chauffe appliquée aux tubes ascendants, on observe, soit la stabilisation du débit autour d'un nouveau régime permanent (après avoir réduit la demande de vapeur), soit au contraire le blocage plus ou moins complet de la circulation.

Un embryon d'étude théorique permet d'établir la condition (suffisante, mais pas nécessaire) de stabilité :

$$
-\frac{\Delta p}{\Delta t} \leqslant w_{f} \gamma \frac{\mathrm{H}}{\mathrm{L}}-\Delta \mathrm{P}_{r}
$$

Dans cette formule, $-\Delta p / \Delta t$ est la vitesse de variation de la pression au-dessus du ballon, $\gamma$ est la densité de l'eau, $\mathrm{H}$ la dénivellation, et $L$ la longueur d'un tube de retour. $P_{r}$ est un terme supplémentaire correspondant aux pertes de charge; $w_{f}$ est la vitesse de circulation dans le tube de retour au début de l'expérience. Cette condition est obtenue en écrivant que, si la vitesse de circulation est suffisante, l'eau dans les tubes de retour subit un accroissement de pression hydrostatique qui compense la baisse de pression au-dessus de la surface libre. Dans ces conditions, il ne peut $y^{\prime}$ avoir vaporisation dans les tubes de retour, et la stabilité de la circulation est assurée.

Les résultats expérimentaux, portés dans le plan $\left(w_{f},-\Delta p / \Delta t\right)$ montrent que la stabilité est obtenue dans un domaine beancoup plus étendu que la région correspondant à (1) (fig. 3). L'écart est considérable, et me paraît provenir

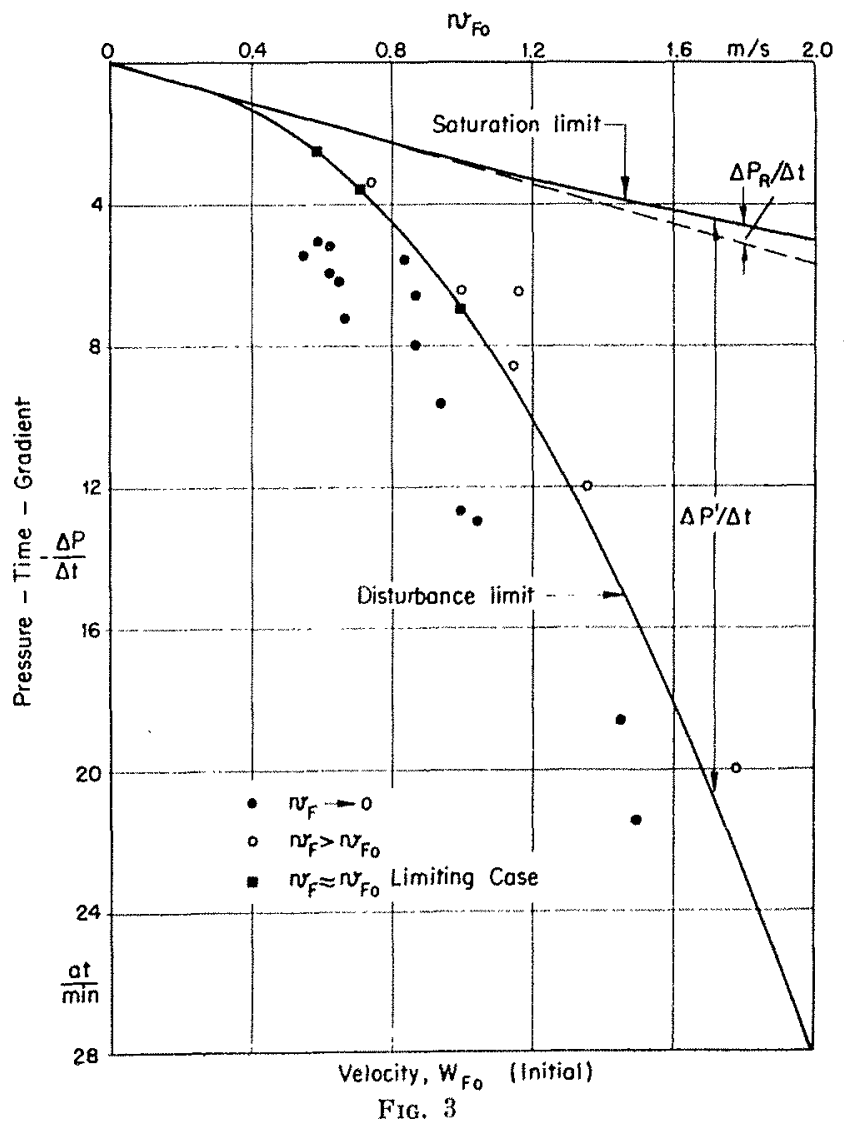


de ce que l'on n'a pas tenu compte ici de l'énergie cinétique de l'eau en circulation. Cette énergie cinétique s'oppose au ralentissement de la circulation, et donne une marge de stabilité supplémentaire.

Sans faire un calcul rigoureux, il est facile de se rendre compte que cette marge a précisément l'ordre de grandeur voulu pour expliquer l'écart.

La preuve me paraît donc faite, après la lecture de ces trois articles, qu'à condition de posséder les caractéristiques hydrauliques et thermiques des différentes parties constitutives d'une chaudière à circulation naturelle, il est possible, par le calcul, de prévoir son comportement en toutes circonstances. Je crois que ce résultat est important et nouveau.

Je passe au septième article de ma liste :

M. W. Garbon, W. D. Gilmert, C. R. Mac Nutt, R. NEIDER :

Steam Gencration in a Reactor. (Nuclear Science and Engineering Congress, Cleveland, preprint 46).

Il s'agit d'une étude expérimentale sur l'ébullition dans l'espace annulaire compris entre un barreau horizontal long de 7,2 $\mathrm{m}$, chauffé électriquement par passage direct de courant continu, et une enveloppe cylindrique concentrique laissant un passage libre de $2,5 \mathrm{~mm}$ d'épaisseur; la cellule est alimentée en circulation forcée. Le barreau utilisé normalement a 7,2 $\mathrm{m}$ de longueur utile, et correspond probablement aux dimensions des canaux des réacteurs de Hanford.

Il peut paraittre bizarre de chercher à faire de l'ébullition sous flux thermique ćlevé dans un tube horizontal; il est bien évident que cette disposition ne favorise pas l'évacuation des poches de vapeur qui peuvent se former. En fait, en lisant attentivement le mémoire, on s'apercoil que le but inilial de l'étude entreprise était de chercher à augmenter, en utilisant l'ébullition, la puissance des piles américaines de Hanford, en vue d'une production accrue de plutonium. Ces piles sont actuellement refroidies par circulation d'cau de rivière.

Les flux thermiques utilisés ici alleignent 180 i 190 watts par $\mathrm{cm}^{2}$. Il faut toulefois noter que ce flux n'a pu être obtenu avec le barreau chauffant normal de $7,2 \mathrm{~m}$, mais seulement avec unc longueur utile de $1 \mathrm{~m}$. Le barreau cst en aluminium, il est creux, el ses extrémités sont connectées à un générateur de courant continu. La paroi du barreau est relativement mince $(1,5 \mathrm{~mm})$, et pour éviter l'écrasement du barreau sous l'effet de la pression extérieure, son intérieur est mis sous pression de gaz inerte.
La paroi extérieure de la cellule est pourvue de nervures qui assurent le centrage du barreau. Comme dans le mémoire de Mumm analysé plus haut, la température de paroi, côté eau, se déduit de la température intérieure du barreau, qu'il est possible de mesurer dans de bonnes conditions. Avec un barreau d'aluminium, la chute de température à travers la paroi du barreau est de quelques degrés seulement.

Les auteurs se sont intéressés surtout à la mesure du titre maximum en vapeur de l'émulsion à la sortie de la cellule. Celui-ci a été étudié en fonction de la pression de vapeur, et pour différentes valeurs du flux calorifique.

Le titre en vapeur est limité par la brûlure du barreau, e'est-à-dire sa fusion locale, suivie de rupture. Cette fusion résulte du passage à travers un régime plus ou moins instable, avec augmentation rapide de l'écart de température entre paroi et émulsion. Il y a deux causes d'instabilité. La première, dont le mémoire ne parle guère, est un phénomène de surface souvent mentionné, mais encore très mal connu :

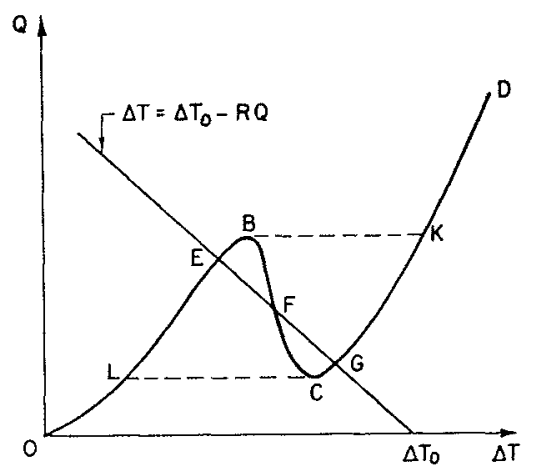

Fig. 4.

Si on porte (fig. 4) en abscisses le $\Delta \mathrm{T}$ en un point quelconque de la paroi, en ordonnces le flux $Q$ correspondant, on obtient une courbe d'allure classique, avec un maximum et un minimum. Dans le cas d'un échangeur lel que $\Delta^{\prime} T$ soil pratiquement imposé, pour chaque $\Delta^{\prime} T$ on obtient un point de fonclionnement el un seul; on peut décrire toule la courbe de maniere réversible. En pratique, la paroi de l'échangeur' introduit tune chute de température $R Q$, et ce qui est imposé, ce n'est pas $\Delta^{\top} \mathrm{T}$, mais l'écart intal $\Delta \mathrm{T}_{1,}$. On a :

$$
\Delta \mathrm{T}=\Delta^{\prime} \mathrm{T}_{\mathrm{n}} \ldots \mathrm{RQ}
$$

Ceci nous définit une droite de fonctionnement, qui se déplace parallèlement à elle-même lorsque $\Delta T_{0}$ varie. Si $R$ est petit, cette droite est presque parallèle à l'axe des ordonnées, et on n'a toujours qu'un seul point de fonctionnement, même sur la branche descendante $\mathrm{BC}$, qu'il est possihle d'explorer complètement. Si R n'est pas petit, 
au contraire, on peut avoir trois points EF G d'intersection. Il est facile de montrer que, dans ce cas, seuls les points extrêmes $\mathrm{E}$ et $\mathrm{G}$ donnent un fonctionnement stable, donc observable.

Il en est de même si, au lieu de chercher à s'imposer la valeur de $\Delta T$, on se fixe le flux thermique $Q$, ce qui est normalement le cas lorsqu'on opère par chauffage électrique. En partant d'un flux faible que l'on fait croitre progressivement, $\Delta \mathrm{T}$ augimente en même temps le long de la branche OB. Arrivé en B, si le flux augmente encore, le point de foncfionmement ne peut que sauter el $K$, et décrit la branche KD. Par flux décroissants, on peut alors décrire la branche DKC, mais en $\mathrm{C}$, le point de fonctionnement saute de nouveau en $\mathrm{L}$, de sorte que la branche $B C$ de la courbe de caléfaction est inobservable. La branche $\mathrm{KD}$ correspond à des valeurs de $\Delta \mathrm{T}$ de l'ordre de plusieurs centaines de degrés, et est déjà difficilement observable avec un barreau chauffant d'aluminium.

La deuxiène cause d'instabilité est due à la nature de l'écoulement, en phase hétérogène: en principe, elle est bien distincte de la premic̀re, mais en pratique, il est certainement très difficile de les distinguer.

Partons d'une certaine valeur du débit-poids. En diminuant ce débit, si l'on avait un écoulement en phase homogène, avec un fluide pas trop dilatable, la perte de charge diminuerait aussi. Mais ici, lorsqu'on diminue le débit, en maintenant constant le flux thermique, le titre en vapeur augmente; donc aussi le volume spécifique de l'émulsion. Il en résulte une vitesse à la sortie plus grande. De sorte qu'il peut très

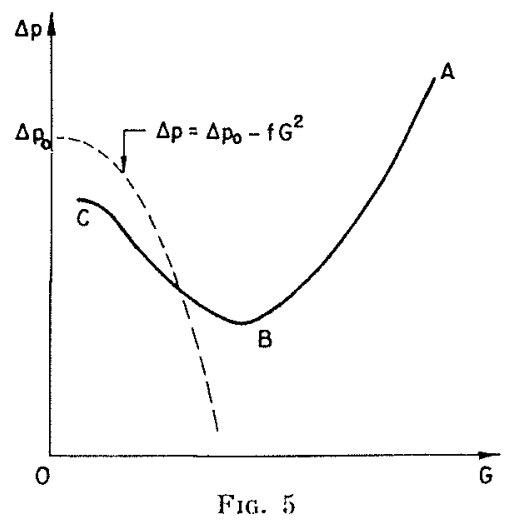

hien arriver -...- il arrive effeclivement -. qu'une réduclion de débit-poids se traduise par une augmentation de la perle de charge tolale. Toul comple fait, la caractéristique perte de charge/ débit a l'allure indiquée figure 5 , avec une branche descendante $\mathrm{CB}$, instable si l'alimentation est faite à la pression quasi-constante. Ici encore, il existe un moyen de stabiliser le système et de décrire réversiblement toute la caractéris- tique : on s'arrange pour disposer d'une pression d'alimentation $\Delta p_{0}$ nettement supérieure à $\Delta p$, et l'on intercale en séric avec la cellule un diaphragme qui cre une perte de charge supplementaire suivant une fonction parabolique du débit. On peut s'arranger pour que la courbe ainsi obtenue :

$$
\Delta p=\Delta p_{0}-f \mathrm{G}^{2}
$$

ne coupe la caractéristique $\mathrm{ABC}$ de la cellule qu'en un seul point, et dans un sens tel que le fonctionnement soit stable. Mais si $\Delta p_{0}$ est fail:le, et l'orifice large, il arrivera gu'on ait deux points d'intersection, dont un seul correspond à un fonctionnement stable.

Le mémoire donne un grand nombre de résultats de mesures. Mais il n'est pas possible de dire dans quels cas les limites de stabilité observées correspondent à l'un ou à l'aulre de ces deux mécanismes : calćfaction vraic, ou instabilité d'écoulement.

De plus, au lieu de se contenter de faire varier la pression de fonclionnement of le flux thermique, les auteurs ont igalement lail varicr la température d'entrée de l'eau dans la cellule, au-dessous de la température de saturation, pratiquant ce que Ies Anglo-Saxons appellent « subcooling ». Les résultals expérimentaux (titre Jimite en fonction de la pression statique, avec le llux calorifique comme parametre) sont reportés en vrac sur le même diagramme, quelle que soit la température d'entrée, ce qui ne facilite pas l'interprétation. Je retiens les conclusions suivantes :

Le titre limite croît à peu près linéairement avec la pression, pour un flux donné, à l'intérieur du domaine exploré. Ceci est tout à fait normal et a déjà été signalé dans le livre de Mac ADAMs.

Il est possible, dans une cellule horizontale -circonstance certainement defavorable -... d'obtenir des lifres en vapeur allant jusqu'à $60 \%$, si on consent a limiter le flux thermique au-dessous de 25 watls/em"

En sens inverse, il est possible de monter jusqu’à 200 watts/cm², si le débil est sulfisanl pour que le tilre de l'énulsion ne dépasse pas $10 \%$.

J'en arrive maintenanl à un huitième et dernier mémoire:

\section{A. R. Gruber et S. C. HYMaN : \\ Flow distribution among parallel heated channels. - Nuclear Science and Engi- neering Congress, Cleveland, Dec. 12-16, 1955, Preprint 349.}

Ce mémoire est de caractere tout it fail thérique, sans aucun résultat expérimental. C'est 
pourtant un des plus intéressants de la séric, bien que plusieurs difficultés donnent l'impression d'y avoir été escamotées.

Le problème étudié est celui de la stabilité de l'écoulement d'un fluide compressible et dilatable, dans un faisceau de tubes alimentés en parallèle à pression constante, en présence d'un flux thermique élevé.

Cette étude s'applique tout naturellement :

- aux réacteurs nucléaires refroidis par gaz;

- aux projets de chaudières fonctionnant audessus de la pression critique.

Les coefficients de convection et de perte de charge utilisés dans les calculs sont obtenus par une méthode due à K. GoldmanN : «Heat Transfer to supercritical water and other fluids with temperature dependent properties » (Chemical Engineering Progress, Symposium series, $\mathrm{n}^{\circ} 11$, vol. 50,1954, p. 105). II s'avère que le paramètre important à considérer est la quantité : $q \mathrm{D}^{0,2} / \mathrm{G}^{0,8}$.

$q$ est le flux thermique local,

D le diamètre hydraulique d'un canal,

G la vitesse massique du fluide.

Le raisonnement suivi n'est malheureusement pas donné avec assez de détails pour qu'on puisse le contrôler. Si nous faisons confiance aux auteurs du mémoire, les résultats sont les suivants, lorsque le flux $q$ varie le long d'un canal suivant un arc de sinusoïde (cas des réac(eurs nucléaires) :

La température en un point donné de la paroi ou du fluide ne dépend que des quatre quantités :

$$
\gamma_{\text {wata }} / \mathrm{Q}, h_{i}, h_{10}, \mathrm{G}^{0.2} \mathrm{D}^{1,2} / \mathrm{L}
$$

$q_{\operatorname{mix}}$ est le flux thermique au contre, $Q$ le flux thermique moyen, $h_{i}$ l'enthalpie du fluide à l'entrée, $h_{0}$ son enthalpie à la sortie; L est la longueur d'un canal.

Les pertes de charge par frottement sont données par une expression de la forme:,

$$
\frac{\Delta p_{f}}{\mathrm{G}^{2}}=\frac{1}{\mathrm{G}^{0,1} \mathrm{D}^{0,1}} \mathrm{~F}\left(h_{i}, h_{0}, \frac{q_{\mathrm{m}: 1, \mathrm{~K}}}{\mathrm{Q}}, \frac{\mathrm{G}^{0,2} \mathrm{D}^{0,2}}{\mathrm{~L}}\right)
$$

La variation $d u$ facteur $1 / G^{0,1} D^{0,1}$ est en pratique négligeable. Pour étudier la stabilité de l'écoulement, il faut déterminer les dérivées partielles :

$$
\mathrm{A}=\left(\frac{\partial \Delta p / \Delta p}{\partial \mathrm{Q} / \mathrm{Q}}\right)_{\mathrm{G}=\mathrm{C} \text { te }} \quad \mathrm{B}=\left(\frac{\partial \Delta p / \Delta p}{\partial \mathrm{G} / \mathrm{G}}\right)_{\mathrm{Q}=\mathrm{Cte}}
$$

La «sensibilité » de l'écoulement est donnée par la connaissance de la quantité :

$$
\mathrm{C}=\left(\frac{\partial \mathrm{G} / \mathrm{G}}{\partial \mathrm{Q} / \mathrm{Q}}\right)_{\Delta p=\mathrm{C}^{\mathrm{te}}} \mathrm{C}=-\frac{\mathrm{A}}{\mathrm{B}}
$$

Avec un fluide dont les propriétés (chaleur spécifique, viscosité, conduction thermique) seraient constantes, on aurait :

$$
\mathrm{A}=0 \quad \mathrm{~B}=1,8 \quad \mathrm{C}=0
$$

Un fluide tel que $C=1$ verrait son enthalpie a la sortie rester constante, quel que soit le flux thermique, ce qui serait l'idéal pour les différents canaux de refroidissement d'un réacteur nucléaire, qui travaillent sous des flux thermiques différents.

Il est parfois possible, avec des fluides réels, d'obtenir une valeur de $C$ légèrement positive, mais il faut plutôt compter, en général, sur une valeur franchement négalive, de sorte que, lorsque le flux thermique varie, le gain d'enthalpie du fluide, entre l'entrée et la sortie (toujours sous pression constante) varie plus que proportionnellement.

L'étude de la température de paroi conduit à des considérations analogues. Si le fluide utilisé est un gaz parfait, on a :

$$
\mu=x^{\prime} \mathbf{T}^{n}
$$

(u. est la viscosite; pour l'air, $n=0,65$ ).

En écoulement laminare, et en ne tenant compte que des pertes de charge par viscosité, on lrouve :

$\mathrm{C}=\frac{(n+1)\left(\mathrm{T}_{0} / \mathrm{T}_{i}\right)^{n+2}-(n+2)\left(\mathrm{T}_{0} / \mathrm{T}_{i}\right)^{n+1}+1}{n\left(\mathrm{~T}_{\mathrm{i}} / \mathrm{T}_{i}\right)^{n+2}-(n+2)\left(\mathrm{T}_{\mathrm{n}} / \mathrm{T}_{i}\right)^{n+1}+2}$

$\mathrm{T}_{0}=$ lempéralure de sortie.

$\mathrm{T}_{i}=$ température d'entrće.

C est négatif, lorsque $\mathrm{T}_{0} / \mathrm{T}_{i}$ est compris entre 1 el 3,7. Lorsque $\mathrm{T}_{0}=3,7 \mathrm{~T}_{i}, \mathrm{C}$ devient infini, l'écoulement devient complètement instable, car loute augmentation du flux thermique fait tendre le débit vers 0 .

En pratique, on a plutôt affaire à des écoulements turbulents, et, dans les mêmes conditions, on trouve :

$$
\mathrm{G}=\frac{-(0,2 n+1)\left(\mathrm{T}_{0} / \mathrm{T}_{i}\right)^{0,2 n+2}+(0,2 n+2)\left(\mathrm{T}_{0} / \mathrm{T}_{i}\right)^{(, 2 n+1}-1}{(0,8-0,2 n)\left(\mathrm{T}_{0} / \mathrm{T}_{i}\right)^{0,2 n+2}+(0,2 n+2)\left(\mathrm{T}_{0} / \mathrm{T}_{i}\right)^{0,2 n+1}-2,8}
$$




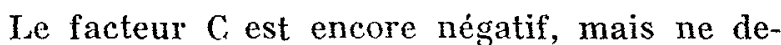
vient infini que si $\mathrm{T}_{0}$ est lui-même infini. Toute variation du flux thermique se répercute, amplifiée, sur la température de sortie, mais il ne peut y avoir arrèt de l'écoulement.

La deuxième partie de l'article étudie les moyens dont on dispose pour réduire ces effets. Le moyen le plus simple est de placer en série avec le canal un diaphragme d'ouverture réglable, à l'entrée ou à la sortie. La méthode la plus efficace pour'stabiliser l'écoulement est d'asservir l'ouverture du diaphragme à la température de sortie. Le calcul montre alors - on pouvait s'en douter - que les meilleurs résultats s'obtiennent en plaçant le diaphragme à l'entrée. Il parait toutefois fort difficile d'aboutir ainsi à une solution mécanique suffisamment robuste et sensible: sans que l'organe de transmission occupe une fraction trop élevée de la section d'écoulement disponible. Une solution plus simple est de placer le diaphragme d'ouverture variable a la sortie. Les performances sont moins bonnes, mais encore trìs acceptables.

Enfin, pour eviter la complication d'un asservissement à la lempérature de sortic, on peut se contenter d'un diaphragme fixe. Le calcul montre qu'il n'y a aucun intérêt à placer un tel diaphragme à la sortie, et qu'il faut le placer à l'entrée. Ce résultat n'est pas nouveau. Il y a déjà longtemps, je crois, que l'on construit des chaudières à circulation forcée du type La Mont. Pour assurer en toutes circonstances la stabilité de la circulation dans le faisceau de tubes connectés en parallèle, ces chaudières sont munies, en tête du faisceau, d'un système d'orifices baptisés tuyères. Ce qui est nourvau ici, c'est d'avoir des données quantitatives. Dans le cas des chaudières La Mont, la théorie ne présentait pas grand interet, car la puissance de pompage y est relalivenent faible, et une augmentation, mêne importante, des pertes de charge n'a pas de rípercussion sérieuse sur le rendement de l'enscmble. Dans un réacteur nucléaire refroidi par gaz, aver les lempératures de paroi actuellemenl admissibles, la puissance de soufflage est au contraire très élevée, et il importe de limiter au plus juste la perte de charge, sans pour autant sacrifier la stabilité de fonctionnement.

Dans une troisicme partie, les auteurs donnent quelques résultats numériques, relatifs à un avant-projet de chaudière super-critique.

Pour : une pression statique de $350 \mathrm{lg} / \mathrm{cm}^{2}$, une température de $260^{\circ} \mathrm{C}$ à l'entrée et $625^{\circ} \mathrm{C}$ à la sortie, un flux thermique de 85 watts $/ \mathrm{cm}^{2}$, un diamètre hydraulique de $1,26 \mathrm{~cm}$ et une longueur de canal de $20 \mathrm{~m}$, on trouve que $\mathrm{C}=-3,75$.

Il en résulte qu'une variation de 1 to du débit provoque une variation de $67^{\circ} \mathrm{C}$ de la tempéralure de sortie.

Une augmentation de $1 \%$ du flux de chaleur augmente de $45^{\circ} \mathrm{C}$ In température maximum de paroi et diminue le débit de $3,75 \%$. Une inégalité de 1 \% sur le diamètre des différents lubes du faisceau se traduit par une différence de $60{ }^{\circ} \mathrm{C}$ cntre les temperatures de sorlic, et une différence de $6,3 \%$ sur les débils. En même temps, la diflérence sur les lempératures de jaroi allaint $70^{\circ} \mathrm{C}$.

Jai également quelques résultals numeriques sur le supplément de perles de charge qu'il faul consentir à travers un diaphraguse placé en tête, pour obtenir une amélioration donnée de la stabilité. Par exemple, pour limiter à $15^{\circ} \mathrm{C}$ Ia variation de temperature de sortic provoquée par une variation de $1 \%$ du flux thermigue, le supplément de pertes de charge à prévoir est. cgal à la perte de charge à la traversce du faisceau. Ceci doit constituer à peu près l'optímum, car, avec une perte de charge infinie, autrement dit en travaillant $\dot{a}$ déhit constant, la tempéralure de sortie augmente encore de $8{ }^{\circ} \mathrm{C}$ pour la même variation de $1 \%$ du flux de chalenx.

Me voici areive au lerme d'un exposé passablement decousu, de par son sujet même. J'aurai atteint mon bul si j'ai pu donner à quelquesuns d'entre vous le désir de se référer aux mémoires que j’ai cilis, qui nont peutêtre pas encore trouve l'audience gu'ils me paraissent mériter.

\section{DIS C USSION}

president: M. GHBirat

M. le Président remercie M. Jour ef owve la diseussion.

Il semble à M. Malese, d'après des expériences récentes a la fois en France, en Grande-Bretagne et aux FtatsUnis, que la notion de diametre hydraulique soit parfaitement applicable dans les caleuls de pertes de charge et également pour les échanges de chalenr même pour des séométries assez complexes. Ceci, bien entendu, à la condition que les coefficients sans dimensions introduits soient correctement definis at que les grandeurs therniques qui $y$ interviennent soient prises à des tempérafures bien déterminées (températures de paroi, température de la masse du fluide, moyenne arithmétique...).

Il serait done intéressant de bien connatre les condifions expérimentales qui ont foumi les résultals cites par le conferencier sur les augmentations des coefficients de pertes de charge et d'echanges de chaleur par rapport aux résultats classiques et de vérifier que les régimes 
étaient bien établis, que les températures ont été correctement mesurées malgré les grandes difficultés possibles, et enfin que les auteurs du rapport ont bien cmployé des définitions correctes.

il. Jouty répond que Mrller, Byinnes et Benforado, dans leurs deux articles, affirment, sur lat foi d'essais effectués avec des barreaux chauffants de longueurs différentes, que le régime permanent hydraulique et thermique était bien établi. Par ailleurs, la mesure des températures est évidemment le point faible de leur travail. Les auteurs ne donnent que fort peu de détails sur la conduite des essais, et l'exploitation des résultats; il est donc difficile de porter un jugement objectif. La tradition en pareil cas est de se fier, jusqu'à preuve du contraire, à la bonne foi et à l'habileté des auteurs. Il serait évidemment souhailable que d'autres expérimentateur's reprennent ces mesures.

Il faut également remarquer (note ajoutée sur éprenves) que l'ésoulement anuulaire utilisé est très mince, $2.75 \mathrm{~mm}$ dépaisseur pour un $\oslash$ extérieur de $21,5 \mathrm{~mm}$, et sa géométrie très éloignée de celle du tube circulaire; alors qu'une barre ailetèe centrée dans un tube (fig. cicontre) délimite une série d'alvéoles donl la section est plus ramassée, et pas tellement difiérente d'un cercle. C'est peut-être pourquoi la notion de diamètre hydraulique s'avère utilisable dans ce dernier cas et pas dans le premicr.

Sur la demande de M. Fonten, M. Joutr précise que les auteurs cités définissent la perte de charge comme la différence de pression entre les cxtrémités du conduit.

Or, M. Fonter signale que cette différence de pression dans le cas d'un lluide de densité variable, n'a pas de rapport avec les forces de frottement le long des parois. C'est probablement pourquoi l'auteur ne retrouve pas les coefficients de frottement classiques. En introduisant une définition correcte de la perte de charge l'expérience montre, comme l'a indiqué $M$. Ls: Folt ce main, que le coefficient de frottement moven ne depend pas de la forme du conduit mais seulement d'un nombre de Reynolds faisant intervenir le rayon hydraulique.

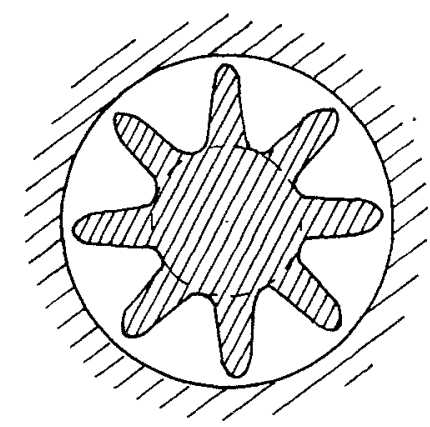

M. Joury répond que les expériences relatées dans le mómoire de Miller, Brrnes, Benforado ont été effecluées en eau sans ébullition, done sur un fluide de densité pratiquement constante.

M. Le Foll confirme la remarque de M. le Professeur Fortren en précisant les valeur's numériques: dans le cns d'un tube circulaire refroidi par de l'nir circulant en régime elabli, la correction à apporter est de l'ordre de l'excès relatif de la température Kelvin du tube sur celle di fluide.

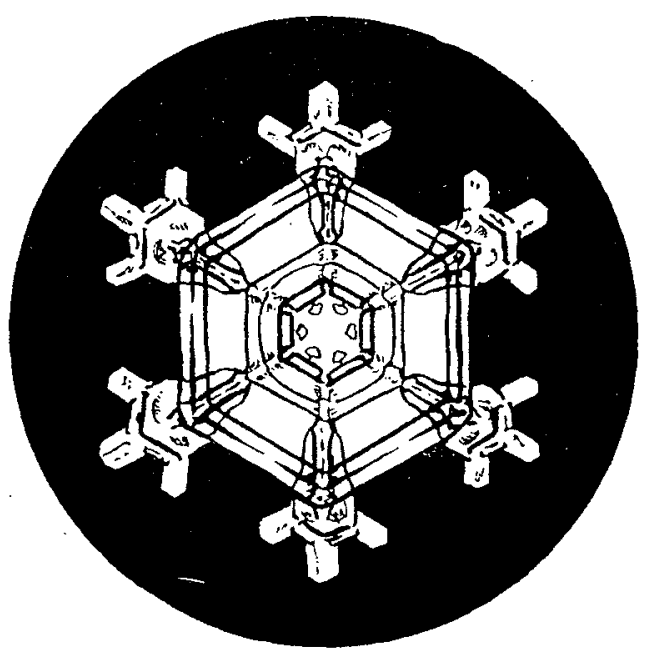

